\title{
Tailoring plasmonic response by Langmuir-Blodgett gold nanoparticle templating for the fabrication of SERS substrates
}

\author{
M. Tahghighi ${ }^{\mathrm{a}, \mathrm{b}}$, I. Mannelli ${ }^{\mathrm{a}}$, D. Janner ${ }^{\mathrm{c}, \mathrm{d}}$, J. Ignés-Mullol ${ }^{\mathrm{a}, \mathrm{b}}$ \\ ${ }^{a}$ Departament de Cincia de Materials i Química Física, Universitat de Barcelona, Martí i Franquès 1, 08028 Barcelona, Spain \\ ${ }^{b}$ Institute of Nanoscience and Nanotechnology (IN2UB), Universitat de Barcelona \\ ${ }^{c}$ Politecnico di Torino, Dipartimento di Scienza Applicata e Tecnologia (DISAT) \\ ${ }^{d}$ INSTM UdR Torino Politecnico, Corso Duca degli Abruzzi 24, Torino 10129, Italy
}

\begin{abstract}
Nanoparticle self-assembly is a robust and versatile strategy for the development of functional nanostructured materials, offering low-cost and scalable methods that can be fine-tuned for many different specific application. In this work, we demonstrate a pathway for the fabrication of tailorable quasi-two-dimensional lattices of gold nanoparticles to be used in Surface Enhanced Raman Scattering (SERS) detection of biomolecules. As a first step, nanoparticles are spread as a monolayer at the water/air interface, compressed to a target lateral density in a Langmuir-Blodgett technique, and transferred to a properly functionalized substrate surface. Once firmly adhered to the substrate, the lattice of nanoparticles can be directly used or be further processed using electroless gold deposition to let the nanoparticle grow thus tuning the plasmonic response and SERS enhancement. Compared to direct deposition or self-assembly methods, our protocol enables to obtain consistent results and much higher coverage of Au nanoparticles thanks to the active control of the surface pressure of the spread monolayer.
\end{abstract}

Keywords: Langmuir-Blodgett, gold nanoparticles, plasmonics, SERS, biosensors.

\section{Introduction}

The development of nanostructured materials based on the self-assembly of simple constituents mediated by wet chemistry methods has been demonstrated to be an efficient and scalable bottom-up strategy[1, 2, 3, 4]. A particularly versatile approach is the use of spherical nanoparticles as building blocks[4]. The existence of well-established synthesis methods to obtain monodisperse batches using a disparity of materials, combined with the inherent tendency of spheres to self-assemble into ordered 2D or 3D lattices, has resulted in a wealth of examples where, depending on the size, material, and surface structure and chemistry, nanoparticles have been employed to build photonic materials[5], functional substrates[6], or biosensors[7], just to name a few.

Among the different methods that have been proposed to control the self-assembly of nanoparticles, Langmuir-Blodgett (LB) deposition[8] stands out. Originally developed for the transfer of insoluble surfactants previously organized at the water/air interface, its use has been extended to the deposition of

Email address: jignes@ub.edu (J. Ignés-Mullol) supramolecular entities[9] and hard nanomaterials[10]. In the LB technique, a hydrophilic substrate of arbitrary shape and wide size range is initially submerged in the water subphase. After the monolayer is spread and adjusted to the desired packing density, the substrate is smoothly pulled through the interface, thus peeling a thin film of water that carries the monolayer with it. Film drainage and water evaporation results in the deposition of the spread monolayer, with preserving the original lateral order.

Structured thin films such as ordered monolayers or multilayers of nanoparticles are particularly well-suited to be prepared by the LB technique, and have been employed for the preparation of photonic materials or as templates to improve the tribological properties of substrates $[11,12]$. Additionally, noble metal nanoparticles are widely used in biosensing thanks to their plasmon resonance that enhances scattered signals of adsorbed biomolecules [13, 14]. Raman spectroscopy is one of the most used for molecule identification and SurfaceEnhanced Raman Scattering (SERS) [15] leverage the plasmonic effects to increase by orders of magnitude the efficiency of an otherwise very inefficient process. The 
realization of reliable and easy to fabricate SERS substrates is of paramount importance for this technique to move from few research lab to a routinary and cheap analysis.

One of the key aspects related to the efficiency of SERS substrates is the presence of hot-spots, places where the light is trapped by a plasmonic effects. Increasing and tuning the hotspots density on a substrate is a fundamental step to control the SERS response. The optimization of the surface density of hotspots, ideally is realized by controlling the spacing and lateral dimensions between nanoparticles which typically are in the range $10-100 \mathrm{~nm}$. A simple, straightforward protocol for the preparation of SERS substrates consists on forming self-assembled monolayers (SAM) of gold or silver nanoparticles in contact with a substrate that has been chemically modified to provide suitable binding sites [16][17]. Available control parameters in this method are the material, size, and shape of the nanoparticles. Due to steric hindrance and the deposition process, the surface coverage obtainable by this technique is limited and in very few cases can reach $50 \%$ of the substrate area reducing a priori the achievable hotspots surface density, although the use of sacrificial organic shells can lead to the preparation of homogeneous hotspot distributions [18]. This method is not restricted to flat substrates, and the use of rough or fibrous substrates offers additional capabilities $[19,20]$. Without having to resort to changes in the particle properties, additional control parameters can be made available by organizing the nanoparticles at the liquid/air interface prior to their transfer to a solid support using the LB technique [21]. Advanced protocols that involve pre- or post-processing of the nanoparticle monolayer have been demonstrated. For instance, nanoparticles can be embedded in a thermosensitive film that allows to tune their spacing [22], and LB deposits can be employed as templates or masks using subsequent lithography techniques in order to manufacture SERS substrated [23]. However, a simple general protocol where the LB films of nanoparticles are directly tuned to optimize their performance as direct SERS substrates has not been demonstrated.

In this work, we use commercial spherical gold nanoparticles to assemble LB films of a controlled lateral density and study their performance to prepare centimeter-scale SERS substrates for the detection of a standard analyte, as a function of the packing density of the nanoparticles[24]. A crucial step in the demonstrated protocol is the proper surface chemistry of the nanoparticles, which must enable their dispersion in a volatile, hydrophobic solvent, and prevent their aggregation that would result in a heterogeneous SERS detec-

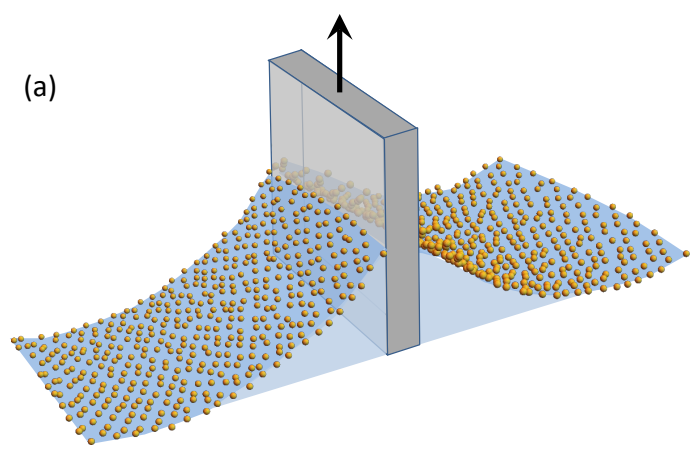

(b)

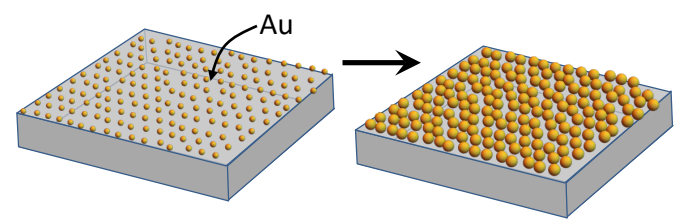

Figure 1: (a) A monolayer of functionalized gold nanoparticles is transferred, at constant surface pressure, onto a hydrophilic plate using the Langmuir-Blodgett technique. (b) The size of the nanoparticles is increased by means of electroless gold deposition.

tion. The performance of the substrate is further tuned by using the transferred nanoparticles as seeds to grow larger gold clusters using a direct electroless protocol, in order to optimize the size of the hotspots.

\section{Experiments}

\subsection{Materials}

Gold nanoparticles (10 $\mathrm{nm}$ in average diameter) were purchased as aqueous solutions from Sigma-Aldrich. 1dodecanthiol (DDT, 98\%), poly(ethyleneglycol)methyl ether thiol $(6000 \mathrm{~g} / \mathrm{mol})$, 4-mercaptobenzoic acid (4MBA, 99\%), hexane $(98.5 \%)$, hydrogen peroxide (30\%), (3-aminopropyl) triethoxysilane (APTES, 99\%), and $\mathrm{HAuCl}_{4}(99.99 \%)$ were purchased from Sigma Aldrich. Ethanol 96\%, sulphuric acid (95\%) and hydrochloric acid (37\%v/v) were purchased from Panreac. Reagent grade toluene was purchased from Scharlau. All chemicals were used as received. Float glass slides (Duran) were used as substrates for UV-vis and SEM characterization, while polished 7980 silica slides where used for SERS measurements. Milli-Q water was used in all suitable processes. 


\subsection{Methods}

\subsubsection{Nanoparticle functionalization}

In the first step, nanoparticles were transferred from the original aqueous solution to a chloroform suspension, suitable for their spreading at the air/water interface. This was achieved by a combination of water soluble PEG-SH and chloroform soluble DDT, as described in Ref[24], which avoids the aggregation that would result from DDT functionalization alone. Briefly, PEG-SH was added to the aqueous particle dispersion to achieve a concentration of 0.8 PEG-SH molecules per $\mathrm{nm}^{2}$ of particle surface, which we have estimated from the nominal particle size and nominal particle concentration reported by the manufacturer. The aqueous solution was put into contact, inside of a test tube, with a DDT chloroform solution with a concentration of 148 DDT molecules per $\mathrm{nm}^{2}$ of particle surface. Vortex shaking at $1500 \mathrm{rpm}$ leads to the particle exchange across the water/chloroform interface. $30 \mu \mathrm{L}$ of concentrated $\mathrm{HCl}$ was added to $10 \mathrm{~mL}$ of nanoparticle solution to assist in the process, which was completed after 30 minutes. The supernatant aqueous phase was replaced with fresh water, and the system was centrifuged for 30 minutes at $4500 \mathrm{rpm}$. This process, which was repeated three times, ensures that the non-adsorbed water-soluble PEG-SH is eliminated. The final result is a suspension of hydrophobic nanoparticles dispersed in chloroform, which will be directly used to spread the monolayers.

\subsubsection{Monolayer preparation and control}

Gold monolayers were spread by dropwise deposition of the chloroform solution onto the water surface in a Langmuir film balance (KSV mini-trough system). Insitu monitoring was achieved by means of a paper Wilhelmy plate pressure balance and a custom made Brewster angle microscope (BAM). After solvent evaporation functionalized gold particles remain at the air/water interface. Careful monolayer compression was performed using the KSV NIMA LB control software. All experiments were performed at room temperature.

\subsubsection{Substrate preparation}

Glass and fused silica slides were modified by silanization with APTES. After cleaning and oxidizing the substrates with piranha solution $(7: 3 \mathrm{v} / \mathrm{v}$ solution of $\mathrm{H}_{2} \mathrm{SO}_{4}$ and $\mathrm{H}_{2} \mathrm{O}_{2}$ ), ethanol, and acetone to remove organic contamination the plates were rinsed with Milli-Q water and finally dried under a nitrogen flow. Clean and dry plates were immersed in an aqueous solution of APTES in toluene $10 \% \mathrm{v} / \mathrm{v}$ at $60^{\circ} \mathrm{C}$ for $120 \mathrm{~min}$. The functionalized plates were rinsed with toluene, methanol and finally with Milli-Q water, and dried under a stream of nitrogen.

\subsubsection{Monolayer transfer onto solid substrates}

Monolayers were transferred at a constant lateral pressure using the Langmuir-Blodgett technique. Plate extraction was carried out at a constant speed of $0.1 \mathrm{~mm}$ $\mathrm{s}^{-1}$ using the dip-coating instrument of the KSV minitrough, which provides a feedback mechanism to maintain a constant pressure in spite of the particle loss during plate extraction.

\subsection{5. vis-NIR espectroscopy}

A Shimadzu UV-1700 spectrophotometer, equipped with a custom 3D-printed holder for the LB-coated plates, was used to characterize the Localized Surface Plasmon Resonance (LSPR) of the different gold films on glass. Spectra from 400 to $800 \mathrm{~nm}$ were acquired at a resolution of $0.5 \mathrm{~nm}$.

\subsubsection{Scannning electron microscopy}

Samples were assembled on the holder by means of a double sided conductive adhesive disk. A thin carbon layer was evaporated on the samples to improve their electric conductivity. Observations were performed with a Jeol J-7100F Field Emission Scanning Electron Microscope.

\subsubsection{Surface-enhanced Raman Scattering}

In order to enhance the Raman signal, nanoparticle monolayers were used as seeds for the electroless deposition of gold [25]. Monolayer-coated plates were soaked in a $10 \mathrm{ml}$ solution of $0.01 \%(\mathrm{w} / \mathrm{v}) \mathrm{HAuCl}_{4}$, and mixed with $500 \mu \mathrm{L}$ of hydrogen peroxide in order to reduce gold chloride ions to atomic gold. The deposition of gold films was conducted under vigorous agitation of the mixture at room temperature for a variable amount of time (2-17 minutes). The resulting gold films were cleaned under $\mathrm{O}_{2}$ plasma to remove traces of organic compounds. Finally, $300 \mu \mathrm{L}$ of a $10 \mu \mathrm{M} 4-\mathrm{MBA}$ aqueous solution (prepared from $1 \mathrm{mM}$ stock solution in ethanol) was incubated on top of the gold substrate for $30 \mathrm{~min}$ to allow a self-assembled monolayer to form, washed with Milli-Q water, and dried under a stream of nitrogen. SERS spectra were obtained within $1 \mathrm{~h}$ of 4MBA monolayer formation using the Raman Dispersive Spectrometer Jobin-Yvon Labram HR 800, coupled to an Olympus BXFM optical microscope. We tested the stability of the films and found no significant variation in the signals $24 \mathrm{~h}$ after 4-MBA incubation at room temperature. The used detector was a 16-bit dynamic range 


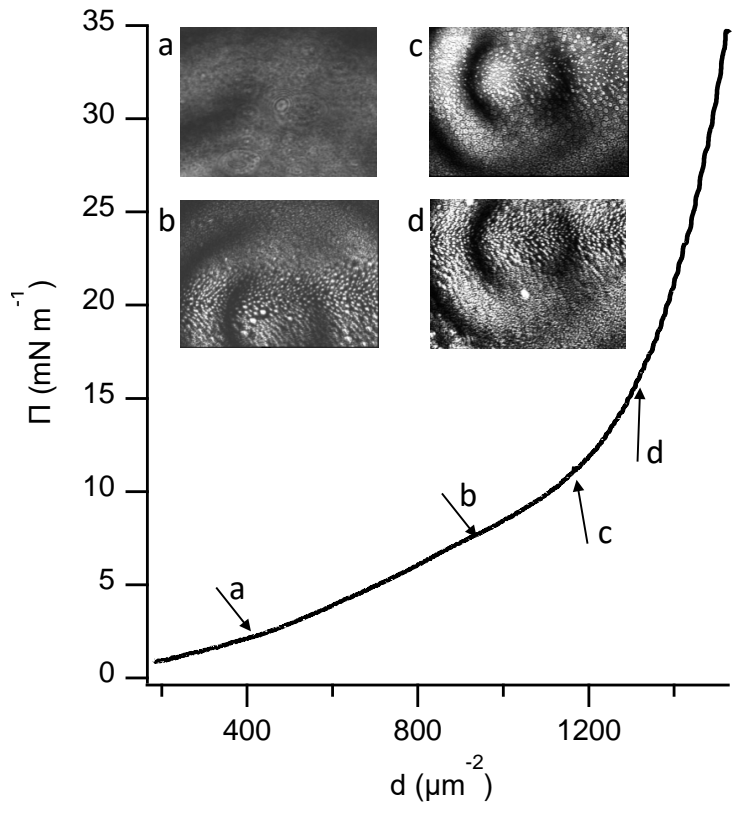

Figure 2: Surface pressure vs. particle surface number density isotherm. Brewster angle microscopy micrographs (field of view is $0.9 \mathrm{~mm}$ wide) have been captured under different compression conditions, as indicated by the corresponding labels.

Peltier cooled CCD. In all the measurements, we illuminated at a wavelength of $532 \mathrm{~nm}$, with a laser power on the sample in the range $0.5-4 \mathrm{~mW}$, using a diffraction grating of 600 lin $\mathrm{mm}^{-1}$ and 100x or 50x microscope objectives. The SERS signal maps were obtained by scanning an area of $25 \times 25 \mu \mathrm{m}^{2}$. The software Labspec (Horiba Ltd.) was used to process SERS data and IgorPro (Wavemetrics, Inc.) to flatten the spectra.

\section{Results and Discussion}

\subsection{Langmuir Monolayers of nanoparticles}

The chloroform suspension of functionalized particles (Sec. 2.2.1), with a nominal concentration of $5.47 \times 10^{16}$ particles $/ \mu \mathrm{L}$, was spread dropwise over the water/air interface using a $50 \mu \mathrm{L}$ Hamilton glass syringe. Milli-Q water in equilibrium with atmospheric $\mathrm{CO}_{2}$ was used as subphase (nominal $\mathrm{pH}=5.7$ ). The initial particle surface concentration was kept low enough to avoid irreversible formation of 3D clusters of nanoparticles. Realtime BAM imaging was employed to monitor the particle self-assembly and uniformity of the resulting film. Under BAM observation, the reflectivity from the thin film increases as the square of its thickness[26], so the formation of thicker patches following excessive film compression is readily detected. Capillarity-

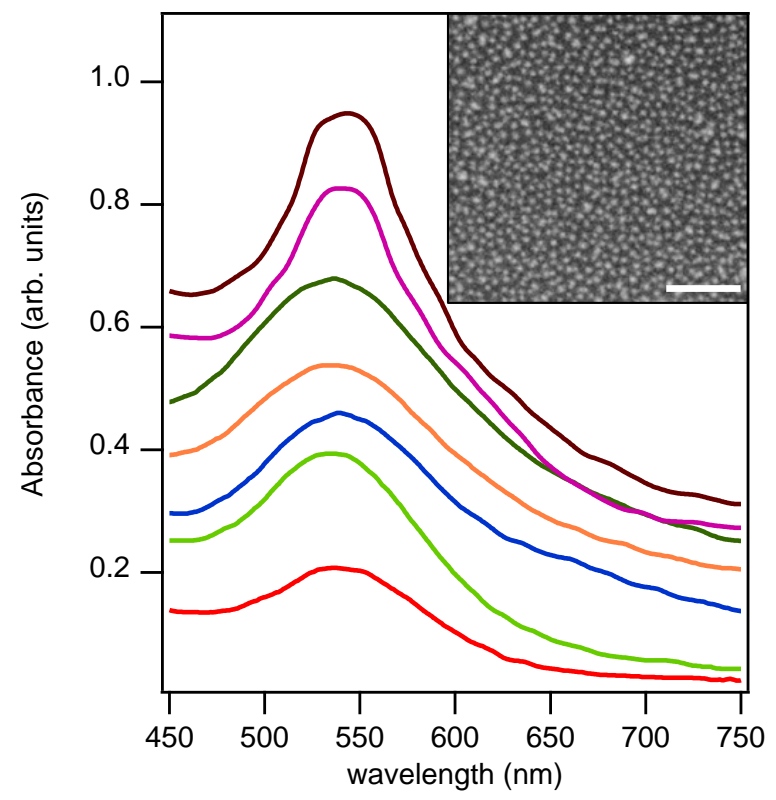

Figure 3: vis-NIR absorption spectra for LB films of $10 \mathrm{~nm}$ gold nanoparticles, prepared under different surface pressures. From bottom to top, $\Pi=3,6,8,10,12,15$, and $17 \mathrm{mN} \mathrm{m}^{-1}$. In the inset, SEM image for a $\mathrm{LB}$ film obtained at $17 \mathrm{mN} \mathrm{m}^{-1}$. The scale is $200 \mathrm{~nm}$.

mediated interparticle interactions result in a direct coupling between lateral packing density and surface pressue (surface tension reduction). Consequently, monitoring of the surface pressure provided with a handle to control the state of the particle monolayer (Fig. 2). Notice that the rise of the surface pressure begins at surface densities as low as 100 particles $/ \mu \mathrm{m}^{2}$, which corresponds to an average center-to-center particle distance of $\sim 100 \mathrm{~nm}$. This observation is consistent with the expected repulsion between electrical dipoles perpendicular to the interface that arise from the electrical double layer that forms around the submerged portion of the particles[27]. Monolayer collapse begins at around $15 \mathrm{mN} \mathrm{m}^{-1}$, as observed from BAM imaging. This provides with an ample range to tune nanostructure of the subsequent LB layer.

Once the film is at the chosen lateral pressure, LB transfer proceeds at constant pressure by means of the feedback mechanism included in the film balance equipment. The hydrophilic plate (glass or fused silica) had been submerged in the subphase prior to particle spreading. While we have silanized these substrates with APTES in order to improve the stability of gold adhesion, we have verified that similar results are achieved on plain substrate provided it is rendered highly hydrophilic as described above. As an alternative to the 


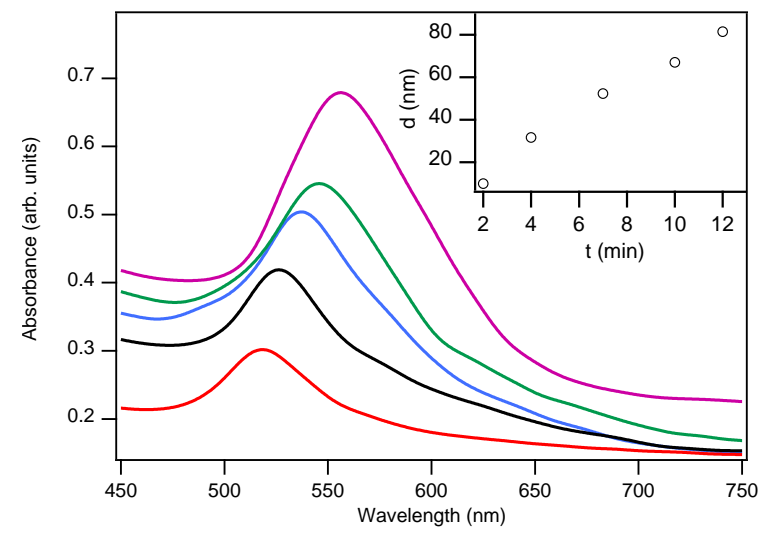

Figure 4: vis-NIR spectra for LB films of 10nm gold nanoparticles prepared at $5 \mathrm{mN} \mathrm{m}^{-1}$ of lateral pressure, and different subsequent gold electroplating time. From bottom to top 2, 4, 7, 10, and $12 \mathrm{~min}$ utes. Inset: average hotspot size estimated from the redshift in the spectra.

LB transfer, the Langmuir-Schaeffer (LS) technique is often employed. In the latter case, the hydrophilic plate is approached, from the air side, onto the interface at a nearly parallel orientation. This technique has the advantage with respect to the LB protocol of a simplified equipment, not needing a surface-pressure with feedback control. A variation of the LS technique is obtained by submerging the hydrophilic plate prior to particle spreading, but keeping it on a flat support. Slow subphase suction leads to the deposition of a monolayer on top of the substrate. While the latter methods have a significant technical simplicity, the LB protocol provides with a potential for better preservation of the lateral structure during transfer, and larger-scale homogeneity of the transferred film.

\subsection{Langmuir-Blodgett films as templates for nanos- tructure formation}

In order to assess the quality of the LangmuirBlodgett films and, in particular, whether significant particle aggregation has occurred, we resort to the measuring the LSPR signal of the surfaces using a standard UV-vis-NIR spectrophotometer. Fig 3a shows the results restricted to the vis-NIR window where plasmon absorption occurs. As expected, the strength of the signal increases for higher surface pressures (higher particle concentration in the measurement windows) but there is no significant redshift in the absorption maxima, except for surface pressure above $15 \mathrm{mN} \mathrm{m}^{-1}$, where the monolayer collapse occurs through the formation of 3D particle clusters. This indicates that particle aggregation is minimized by the proposed preparation protocol. A high resolution SEM micrograph of a LB film obtained just above the collapse pressure (Fig 3b) illustrates the highest achievable nanoparticle density and the incipient formation of 3D aggregates. From the SEM image, we compute a limiting surface number density of $1.2 \times 10^{2} \mu \mathrm{m}^{-2}$. This value is lower than the nominal value estimated from the isotherm (Fig. 2), which can be attributed to the incipient 3D particle aggregation.

The potential for these monolayers to serve as SERS substrates requires a fine tuning of the lattice of hotspots constituted by gold nanoparticles or nanoclusters. Beside the choice of parameters during Langmuir monolayer formation, one can additionally use the lattice of nanoparticles as a seed layer to grow nanostructures of different size and shape[28,29] by means of electroless gold growth[25]. By adjusting the reaction time, we can tune the size, and thus the available surface density of the hotspots. An initial LSPR analysis (Fig. 4) reveals the steady red shift of the absorption maxima, which correlates with an increase in the average nanoparticle size, consistent with the seed growth. We have estimated the average hotspot size by comparing the LSPR redshift in Fig. 4 with calibration data provided by the nanoparticle manufacturer (see inset in Fig. 4).

In these experiments, LB films have been obtained at a lateral pressure of $5 \mathrm{mN} \mathrm{m}^{-1}$, which corresponds to a nominal density of about 70 particles $/ \mu \mathrm{m}^{2}$. SEM images reveal that the arrangement of the particles is heterogeneous (Fig. 5), which is likely a result of the LB extraction and subsequent drying of the aqueous wetting film, responsible for the interparticle repulsion at the water/air interface. A SEM image obtained at a tilted incidence allows to assess the absence of particle multilayer, and to observe the development of a rough surface. Although the LSPR studies on raw LB films (Fig. 3 ) suggested that particle aggregation was not important, gold electroplating will, eventually, build bridges between adjacent particles, resulting in a decrease in the density of hotspots.

\subsection{SERS performance of the gold monolayers}

In order to study the influence of the different control parameters on the performance of these nanostructured surfaces as SERS substrates, we have chosen 4MBA as the target analyte. This molecule chemisorbs to the gold surface with stable covalent bonds, thus benefiting from the amplifying plasmonic effect during Raman scattering measurements. Fig. 6a shows a family of SERS spectra obtained from 4-MBA adsorbed on gold nanoparticle susbtrates prepared with the LB technique at different lateral pressures, followed by a common electroplating time of 8 minutes. We monitor the 

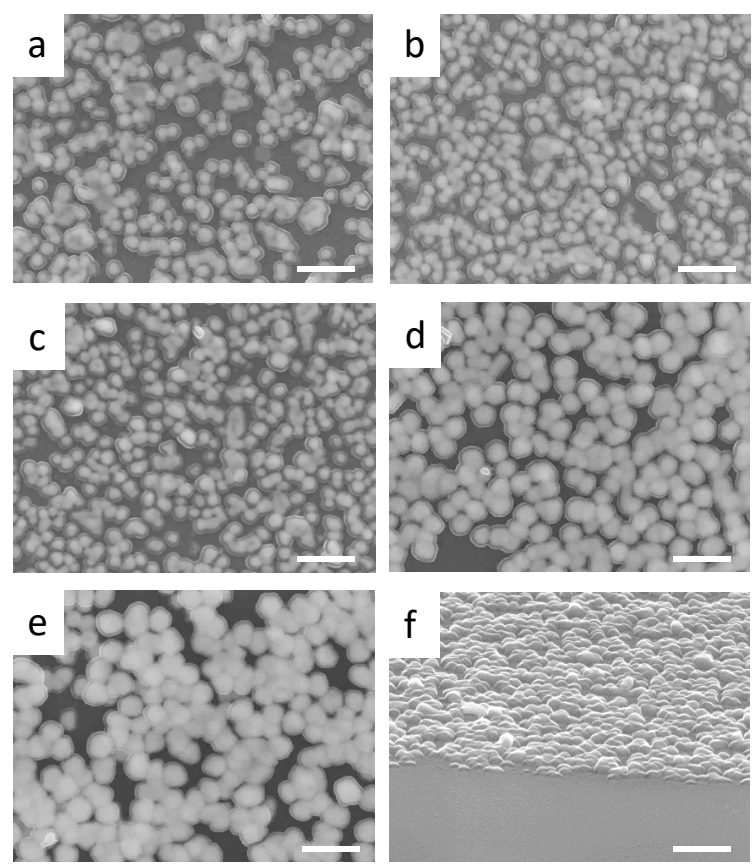

Figure 5: SEM micrographs of nanoparticle LB films after different gold electroplating time, from (a) to (e), $2 \mathrm{~min}, 4.5 \mathrm{~min}, 8 \mathrm{~min}, 10 \mathrm{~min}$, and $12 \mathrm{~min}$, respectively. LB films had been transferred at $8 \mathrm{mN} \mathrm{m}^{-1}$. (f) Tilted image of the same substrate in (e). Scale bars are $500 \mathrm{~nm}$ long.

two well-known main peaks for 4-MBA at $1076 \mathrm{~cm}^{-1}$ and $1588 \mathrm{~cm}^{-1}$, while we filter the rest of the spectrum using bandpass filters. We observe that the SERS intensity increases monotonically for both peaks as the lateral pressure (thus, the particle density) increases, until reaching a maximum followed by a decay in the detection performance. A plot of the peak intensities $v s$. lateral pressure of the LB transfer (Fig. 6b) clearly reveals a pronounced maximum at a pressure around 13 $\mathrm{mN} \mathrm{m}{ }^{-1}$, which corresponds to the limit of monolayer stability. Further compression resulted in the beginning of monolayer collapse, which led to the formation of multilayer aggregates, and the progressive decrease in hotspot density. The shape of the SERS peak intensity vs. lateral pressure hints at the appearance of a resonance at a well-defined packing density. However, it could also be interpreted as a degradation of the substrate performance due to the formation of multilayer patches. A similar trend is observed when we compute the integrated peak areas (Fig. 6c), which may allow for an enhanced SERS sensitivity.

The results are perhaps more clear when we study the performance of substrates prepared at a common lateral pressure of $12 \mathrm{mN} \mathrm{m}^{-1}$ (within the limits of monolayer
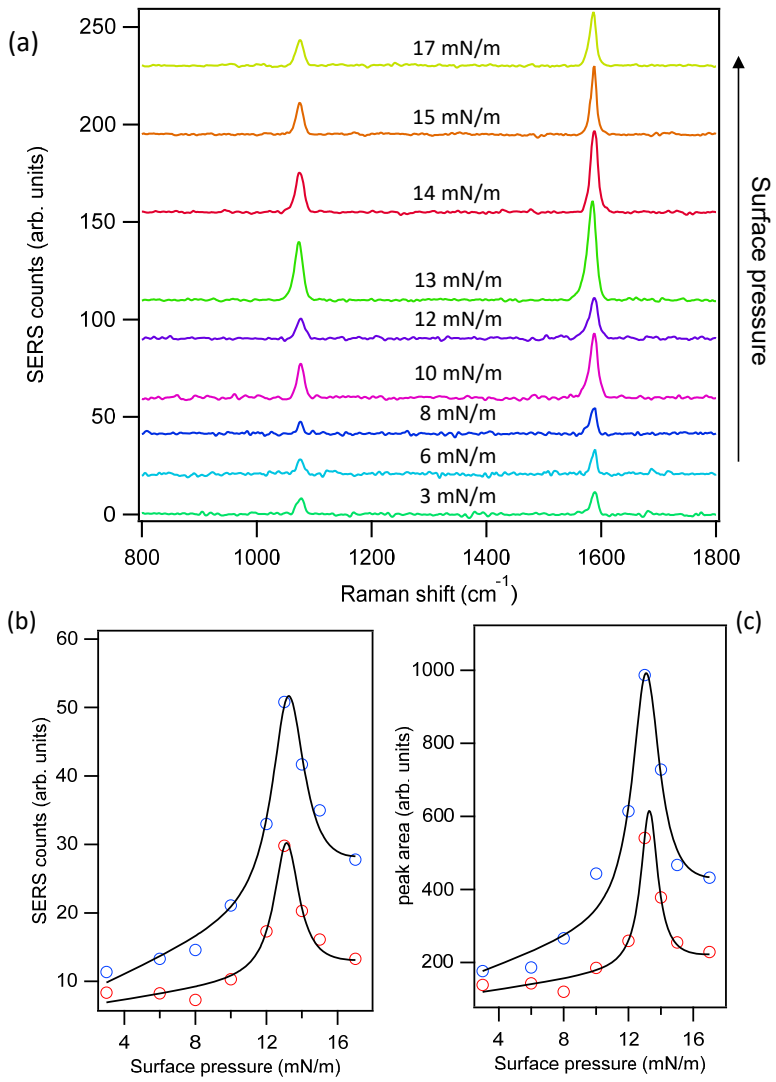

Figure 6: (a) SERS spectra of 4-MBA adsorbed on gold nanoparticle monolayers transferred at different lateral pressures, followed by an electroplating time of 8 minutes. (b) Height of the peaks at $1076 \mathrm{~cm}^{-1}$ and $1588 \mathrm{~cm}^{-1}$. (c) Area of the peaks at $1076 \mathrm{~cm}^{-1}$ and $1588 \mathrm{~cm}^{-1}$. The lines through the data are guides for the eye. 

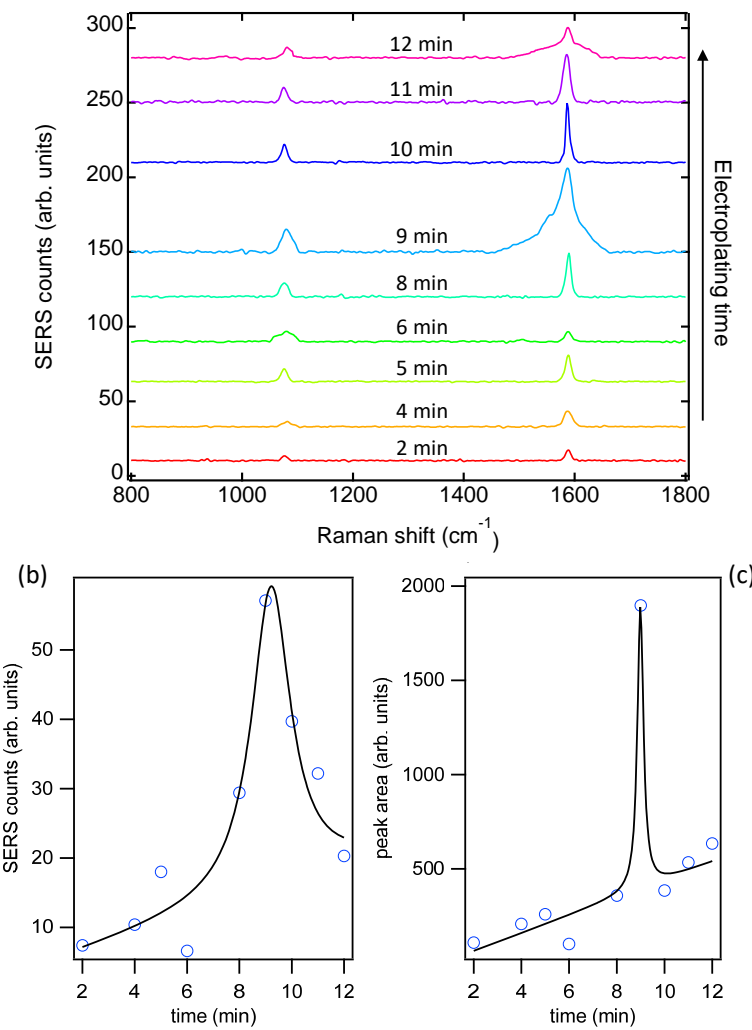

Figure 7: (a) SERS spectra of 4-MBA adsorbed on gold nanoparticle monolayers transferred at a lateral pressure of $12 \mathrm{mN} \mathrm{m}^{-1}$, followed by different electroplating times. (b) Height of the peaks at $1588 \mathrm{~cm}^{-1}$. (c) Area of the peaks at $1588 \mathrm{~cm}^{-1}$. The lines through the data are guides for the eye. stability) as a function of electroplating times (Fig. 7). Data suggest the presence of a resonance at around 9 minutes of electroplating time. Analogous results are obtained when either the peak maxima or the integrated peak intensities are considered (Fig. 7a,b). Although the spike observed in the integrated intensities is remarkable, the shape of the peaks is more sensitive to spurious signals than its maximum, and these data are somewhat less amenable to further study. LSPR data in Fig. 4 indicates that the effective hotspot size is around $60 \mathrm{~nm}$ for this electroplating time. In a forthcoming study we aim at studying the possibility of improving the SERS sensitivity by using monolayers prepared with nanoparticles of larger diameter than those used in the present study. Although an optimum process time should be expected for our substrates, since an excessive electroplating would lead to the progressive erosion of the nanoscale roughness, the fact that a resonant-like behavior is observed demands further experimental work and theoretical modeling.

Finally, we have assessed the homogeneity of hotspot distribution in these nanostructured substrates by mapping the integrated SERS intensity for the $1588 \mathrm{~cm}^{-1}$ 4-MBA peak (from 1530 to $1625 \mathrm{~cm}^{-1}$ ) in a region of $15 \times 15 \mu \mathrm{m}^{2}$, at $1 \mu \mathrm{m}$ steps (Fig. 8). We observe a pseudo-random pattern in the distribution of SERS intensity with a dispersion of $8 \%$. Such dispersion can be caused by the disordered particle assembly (Fig. 5), inhomogeneities in the electroplating process, or simply by the presence of traces of other organic chemicals.

\section{Conclusions}

We have demonstrated a facile protocol for the preparation of fine-tuned SERS substrates based on Langmuir-Blodgett films of gold nanoparticles. Waterbased particles are readily capped with a combination of commercially available organothiols, allowing to disperse them in a volatile solvent that enables to spread the nanoparticles at the water/air interface minimizing unwanted aggregation. We have demonstrated that, for a given particle size, the enhancement for SERS detection of a referent analyte, 4-MBA, can be tuned by controlling the packing density of the nanoparticles at the water/air interface and the thickness of a subsequent gold layer that can be deposited on the LB film using electroless plating. Both protocols reveal the emergence of a resonance in the SERS intensity at welldefined nanoparticle lateral density or gold electroplating, whose physical origin is yet unclear and should be further studied. Combined with custom-synthesized 


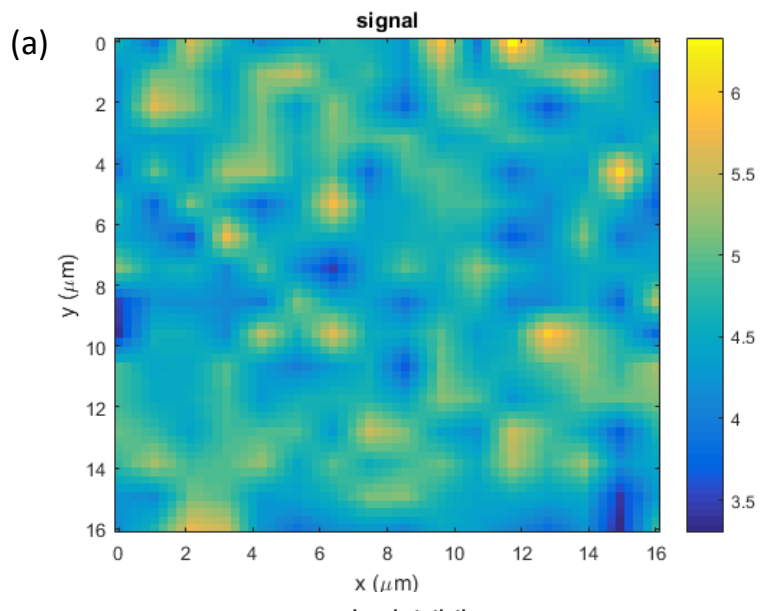

(b)

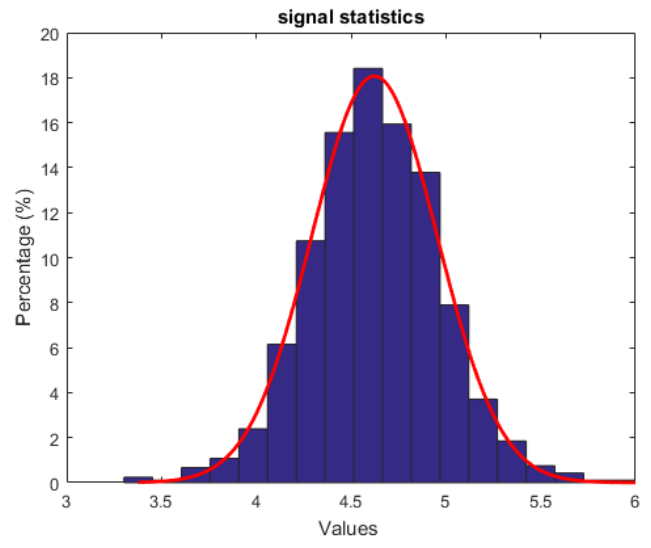

Figure 8: (a) SERS scan of the $1077 \mathrm{~cm}^{-1}$ peak in a sample of 4MBA adsorbed on a gold nanoparticle monolayer transferred at a lateral pressure of $15 \mathrm{mN} \mathrm{m}^{-1}$ and an electroplating time of $8 \mathrm{~min}$. Data has been acquired with the 50x microscope objective. (b) SERS intensity distribution in the $15 \mu \mathrm{m} \times 15 \mu \mathrm{m}$ region shown in (a). The solid line is a gaussian fit giving an average intensity 4.4 (arb. units) and a standard deviation 0.7 (arb. units).

nanoparticles of arbitrary size and shape, the demonstrated protocols could offer a wealth of tuning capacities to optimize the performance of a SERS-based chemical sensor.

\section{Acknowledgements}

J.I-M. and M.T. acknowledge support from MINECO, project FIS2016-78507-C2-1-P. M.T. acknowledges a fellowship from IN2UB. D.J. aknowledges partial support from Compagnia di San Paolo through the Starting Grant Program. All the authors acknowledge funding from NATO project G5250.

[1] G. M. Whitesides, B. Grzybowski, Self-assembly at all scales, Science 295 (5564) (2002) 2418-21.
[2] S. Mann, Self-assembly and transformation of hybrid nanoobjects and nanostructures under equilibrium and nonequilibrium conditions, Nat Mater 8 (10) (2009) 781-92.

[3] J. Zhang, Y. Li, X. Zhang, B. Yang, Colloidal self-assembly meets nanofabrication: from two-dimensional colloidal crystals to nanostructure arrays, Adv Mater 22 (38) (2010) 4249-69.

[4] N. Vogel, M. Retsch, C. A. Fustin, A. Del Campo, U. Jonas, Advances in colloidal assembly: the design of structure and hierarchy in two and three dimensions, Chem Rev 115 (13) (2015) 6265-311.

[5] W. Zhou, M. Dridi, J. Y. Suh, C. H. Kim, D. T. Co, M. R. Wasielewski, G. C. Schatz, T. W. Odom, Lasing action in strongly coupled plasmonic nanocavity arrays, Nat Nanotechnol 8 (7) (2013) 506-11.

[6] O. D. Velev, S. Gupta, Materials fabricated by micro- and nanoparticle assembly - the challenging path from science to engineering, Advanced Materials 21 (19) (2009) 1897-1905.

[7] J. M. Pingarrn, P. Yez-Sedeo, A. Gonzlez-Corts, Gold nanoparticle-based electrochemical biosensors, Electrochimica Acta 53 (19) (2008) 5848-5866.

[8] M. C. Petty, Langmuir-Blodgett films : an introduction, Cambridge University Press, Cambridge ; New York, 1996.

[9] K. Ariga, Y. Yamauchi, T. Mori, J. P. Hill, 25th anniversary article: what can be done with the langmuir-blodgett method? recent developments and its critical role in materials science, Adv Mater 25 (45) (2013) 6477-512.

[10] S. Acharya, J. P. Hill, K. Ariga, Soft langmuir-blodgett technique for hard nanomaterials, Advanced Materials 21 (29) (2009) 2959-2981.

[11] S. Reculusa, S. Ravaine, Synthesis of colloidal crystals of controllable thickness through the langmuirblodgett technique, Chemistry of Materials 15 (2) (2003) 598-605.

[12] S. Portal-Marco, M. A. Vallve, O. Arteaga, J. Ignes-Mullol, C. Corbella, E. Bertran, Structure and physical properties of colloidal crystals made of silica particles, Colloids and Surfaces a-Physicochemical and Engineering Aspects 401 (2012) 38-47.

[13] S. Eustis, M. A. el Sayed, Why gold nanoparticles are more precious than pretty gold: noble metal surface plasmon resonance and its enhancement of the radiative and nonradiative properties of nanocrystals of different shapes, Chem Soc Rev 35 (3) (2006) 209-17.

[14] P. K. Jain, K. S. Lee, I. H. El-Sayed, M. A. El-Sayed, Calculated absorption and scattering properties of gold nanoparticles of different size, shape, and composition: applications in biological imaging and biomedicine, J Phys Chem B 110 (14) (2006) 7238-48.

[15] K. C. Bantz, A. F. Meyer, N. J. Wittenberg, H. Im, O. Kurtulus, S. H. Lee, N. C. Lindquist, S. H. Oh, C. L. Haynes, Recent progress in sers biosensing, Phys Chem Chem Phys 13 (24) (2011) 11551-67.

[16] C. Wang, Y. Xu, H. Zhao, C. Gang, C. Lai, X. Liao, R. Wang, Detection and analysis of sers effect of nano gold by selfassembly chemical plating composite method, Applied Surface Science 353 (2015) 750-756.

[17] Q. Guo, M. Xu, Y. Yuan, R. Gu, J. Yao, Self-assembled largescale monolayer of au nanoparticles at the air/water interface used as a sers substrate, Langmuir 32 (18) (2016) 4530-7.

[18] L. Osinkina, T. Lohmller, F. Jckel, J. Feldmann, Synthesis of gold nanostar arrays as reliable, large-scale, homogeneous substrates for surface-enhanced raman scattering imaging and spectroscopy, The Journal of Physical Chemistry C 117 (43) (2013) 22198-22202.

[19] J. Liu, J. Zhou, B. Tang, T. Zeng, Y. Li, J. Li, Y. Ye, X. Wang, Surface enhanced raman scattering (sers) fabrics for trace analysis, Applied Surface Science 386 (2016) 296-302. 
[20] Z. Liu, Z. Yan, L. Jia, P. Song, L. Mei, L. Bai, Y. Liu, Gold nanoparticle decorated electrospun nanofibers: A 3d reproducible and sensitive sers substrate, Applied Surface Science 403 (2017) 29-34.

[21] A. Tao, F. Kim, C. Hess, J. Goldberger, R. He, Y. Sun, Y. Xia, P. Yang, Langmuirblodgett silver nanowire monolayers for molecular sensing using surface-enhanced raman spectroscopy, Nano Letters 3 (9) (2003) 1229-1233.

[22] Y. Lu, G. L. Liu, L. P. Lee, High-density silver nanoparticle film with temperature-controllable interparticle spacing for a tunable surface enhanced raman scattering substrate, Nano Lett 5 (1) (2005) 5-9.

[23] N. Marquestaut, A. Martin, D. Talaga, L. Servant, S. Ravaine, S. Reculusa, D. M. Bassani, E. Gillies, F. Lagugne-Labarthet, Raman enhancement of azobenzene monolayers on substrates prepared by langmuir-blodgett deposition and electron-beam lithography techniques, Langmuir 24 (19) (2008) 11313-21.

[24] A. B. Serrano-Montes, D. J. de Aberasturi, J. Langer, J. J. GinerCasares, L. Scarabelli, A. Herrero, L. M. Liz-Marzan, A general method for solvent exchange of plasmonic nanoparticles and self-assembly into sers-active monolayers, Langmuir 31 (33) (2015) 9205-13.

[25] J. Hu, W. Li, J. Chen, X. Zhang, X. Zhao, Novel plating solution for electroless deposition of gold film onto glass surface, Surface and Coatings Technology 202 (13) (2008) 2922-2926.

[26] J. Ignes-Mullol, J. Claret, F. Sagues, Star defects, boojums, and cardioid droplet shapes in condensed dimyristoylphosphatidylethanolamine monolayers, Journal of Physical Chemistry B 108 (2) (2004) 612-619.

[27] E. J. Stancik, M. J. O. Widenbrant, A. T. Laschitsch, J. Vermant, G. G. Fuller, Structure and dynamics of particle monolayers at a liquidliquid interface subjected to extensional flow, Langmuir 18 (11) (2002) 4372-4375.

[28] J. He, Y. Wang, Y. Feng, X. Qi, Z. Zeng, Q. Liu, W. S. Teo, C. L. Gan, H. Zhang, H. Chen, Forest of gold nanowires: a new type of nanocrystal growth, ACS Nano 7 (3) (2013) 2733-40.

[29] A. La Porta, M. Grzelczak, L. M. Liz-Marzan, Gold nanowire forests for sers detection, ChemistryOpen 3 (4) (2014) 146-51. 